\title{
Kann der ASD Kinder- und Jugendstärkungsgesetz?
}

\author{
Blitzlichter auf die SGB VIII-Reform aus der Perspektive des ASD
}

Dem Allgemeinen Sozialen Dienst der Jugendämter kommt im System der Kinder- und Jugendhilfe eine besondere Bedeutung zu. In der Folge sind auch die mit dem KJSG in Kraft getretenen Weiterentwicklungen der Kinder- und Jugendhilfe für die Arbeit im ASD besonders beachtenswert. Zugleich hat der ASD eine besondere Rolle in der Umsetzung und Realisierung dieser Neuerungen. Der folgende Beitrag geht der Frage nach, welche An- und Herausforderungen sich dabei für den ASD ergeben und zeigt weitere Handlungs- und Klärungsbedarfe auf.

$\mathrm{E}$ s wurde hektisch rund um den 7. Mai 2021 in den Jugendämtern Deutschlands: Nur die Optimistischsten hatten erwartet, dass Bundestag und Bundesrat sich nach fast fünfjähriger Debatte auf der Zielgerade dieser Legislaturperiode (denn auch in der vorherigen war die SGB VIII-Reform ja bereits anvisiert) auf dieses komplexe und umfangreiche Reformwerk des Kinder- und Jugendstärkungsgesetzes (KJSG) einigen würden. Ein Gesetz, das dem Allgemeinen Sozialdienst (ASD) veränderte und neue Leistungen, detaillierte Verfahrensanweisungen, aber auch in Teilen eine neue Programmatik in die Auftragsbücher schrei-

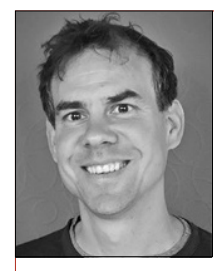

\section{Stefan Pietsch}

Eschweiler, Deutschland

*1975, Dipl.-Sozialarbeiter, Stellv. Jugendamtsleiter und Leiter der Abteilung Soziale Dienste beim Jugendamt der Stadt Eschweiler, Mitglied im Beirat der Bundesarbeitsgemeinschaft ASD.

stefan.pietsch@eschweiler.de

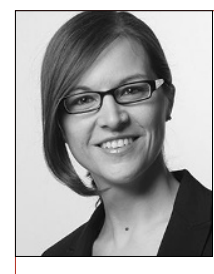

\section{Verena Klomann}

Hochschule Darmstadt, Darmstadt, Deutschland *1977, Dr., Dipl.-Sozialpädagogin (FH), M. A., Supervisorin (DGSv), Professorin für Theorien der Sozialen Arbeit an der Hochschule Darmstadt, Fachbereich Soziale Arbeit, Mitglied in der Bundesarbeitsgemeinschaft ASD.

verena.klomann@h-da.de

\section{Zusammenfassung Zahlreiche Neuerungen der SGB} VIII-Reform wirken sich auf die Arbeit im ASD des Jugendamtes aus. Gleichzeitig wird deren Umsetzung maßgeblich durch den ASD beeinflusst. Exemplarisch werden hiermit einhergehende An- und Herausforderungen aus ASD-Perspektive diskutiert und Entwicklungsimpulse aufgezeigt.

Schlüsselwörter ASD des Jugendamtes, SGB VIII-Reform, Kinderschutz, Hilfeplanung, Inklusion ben wird. Diese Auftragsbücher sind bereits jetzt gut gefüllt und müssten bereits aus der Vergangenheit den ein oder anderen Auftrag stärker in den Fokus rücken. Hinzu kommt eine gesamtgesellschaftliche Auftragslage, die gerade in den letzten Jahren hohe Ansprüche an die Auftragsbearbeitung - wie bspw. hinsichtlich der Begleitung von Menschen mit Fluchtbiografie, im Kinderschutzkontext oder durch die Coronapandemie - gestellt hat.

Kann der ASD also Kinder- und Jugendstärkungsgesetz? Dieser Beitrag beleuchtet diese Fragestellung, gibt zudem einen Rückblick auf den Prozess und beschreibt Potenziale, Hürden und Kritikpunkte im Hinblick auf das KJSG.

\section{Von nicht autorisierten Arbeitsentwürfen und anderen Aufregern}

Ab 2016 gehörte das Aufrufen der Informationssammlung zum SGB VIII-Reformprozess auf der Homepage des Deutsches Institut für Jugendhilfe und Familienrecht e. V. (DIJuF) zur fast täglichen Standardlektüre in den Jugendämtern. Weitergegebene Powerpoint-Folien, nicht autorisierte Arbeitsentwürfe und zuletzt ein Gesetzesentwurf zu einem Kinder- und Jugendstärkungsgesetz machten die Runde, wurden kommentiert und oft inhaltlich kritisiert. Konfliktlinien (z. B. Einzelfallhilfe vs. Sozialraumorientierung) und Ausgestaltungsschwierigkeiten (z. B. zu einem einheitlichen Leistungstatbestand von Hilfen zur Erziehung und Eingliederungshilfen) wurden deutlich. Am 22.09.2017 erfolgte die Absetzung des KJSG von der Tagesordnung des Bundesrates und damit zunächst ein jähes Ende der aufgeregten Diskussion.

Geprägt von diesen Erfahrungen wählte das Bundesministerium für Frauen, Senioren, Familie und Jugend 
(BMFSFJ) mit dem Format „Mitreden - Mitgestalten“ einen neuen Weg, der mit der Auftaktveranstaltung zum 06.11.2018 startete und mit der Abschlusskonferenz zum 10.12.2019 endete. In der zentralen Arbeitsgruppe war der ASD dabei nur indirekt über Vertreter_innen der Kinder- und Jugendhilfe beteiligt. Die Bundesarbeitsgemeinschaft ASD (kurz BAG ASD) wurde bei der Zusammenstellung dieser Arbeitsgruppe durch das BMFSFJ nicht berücksichtigt. Viele Leitungs- und Fachkräfte aus dem ASD nutzten allerdings die Beteiligungsmöglichkeiten im Rahmen der Online-Formate und aus zahlreichen fachspezifischen Konferenzen und Tagungen flossen immer wieder Impulse in den Prozess ein. In der wissenschaftlichen Begleitforschung des Instituts für Kinder- und Jugendhilfe (IKJ) wurde deutlich, dass sich nicht alle identifizierten Handlungsbedarfe in der Kinder- und Jugendhilfe auf gesetzliche Änderungen beziehen bzw. hierdurch beheben lassen. Vielmehr wurden auch Vollzugs- bzw. Umsetzungsdefizite festgestellt. Im Reformprozess sollte deshalb geprüft werden, inwieweit durch eine Schärfung und/oder Ergänzung bestehender Regelungen die Wahrscheinlichkeit einer gelingenden Umsetzungspraxis erhöht werden kann (BMFSFJ 2021b, S. 84).

Nach der Abschlusskonferenz nahm die letzte Etappe des Reformprozesses zum 05.10.2020 mit einem ersten Referentenentwurf Fahrt auf und erreichte im Frühjahr 2021 ihren Abschluss. Dabei wäre auch eine Vollbremsung kurz vor dem Ziel nicht ausgeschlossen gewesen. Letztendlich konnten aber zwischen Bundesregierung und den Bundesländern die notwendigen Kompromissoptionen gefunden werden und so verkündete Familienministerin Franziska Giffey am 10.05.2021: „Es ist ein wahrer Meilenstein: Der Bundesrat hat heute die Reform des Kinder- und Jugendhilferechts beschlossen. Mit dem neuen Kinder- und Jugendstärkungsgesetz verbessern wir die Rechte aller Kinder und Jugendlichen - von der Kita über die Schulsozialarbeit bis hin zu den Pflegefamilien. Es ist die größte Reform seit über 30 Jahren, seit der Einführung 1990.“ (BMFSFJ 2021a).

\section{Der ASD: bunt, unterschiedlich}

\section{und nirgendwo gleich?!}

Die Organisation und inhaltliche Ausgestaltung des ASD als kommunalem Basisdienst sind in den Jugendämtern sehr unterschiedlich. Dies ist zunächst durch die grundsätzliche Unterschiedlichkeit der Jugendämter zu erklären: Organisatorisch sind diese in Deutschland zu 52,3 \% Landkreisen, zu 29,1 \% einer kreisangehörigen Stadt und zu 18,9 \% einer kreisfreien Stadt zugeordnet (vgl. Bundesarbeitsgemeinschaft der Landesjugendämter \& Müller 2020, S. 88). Eine Vielzahl der Jugendäm- ter - meistens bei kreisangehörigen Kommunen - befindet sich dabei in Nordrhein-Westfalen: Hier sind alleine 186 der 559 Jugendämter in Deutschland angesiedelt. Dementsprechend differieren auch die Leistungsspanne bzw. das Aufgabenfeld der Allgemeinen Sozialen Dienste. Sozio- und infrastrukturelle Rahmenbedingungen, die Finanzkraft der jeweiligen Kommune und natürlich auch die jeweiligen kinder- und jugendpolitischen Schwerpunkte sind weitere Einflussfaktoren auf diese Organisationseinheit (vgl. ebd., S. 83 ff.). Durchzusetzen scheint sich aber - anders als noch zur Zeit der Einführung des KJHG - eine grundsätzliche Ausrichtung der Allgemeinen Sozialdienste auf die Kinder- und Jugendhilfe und damit eine Reduktion des „Allgemeinen“ auf diese Zielgruppe. Trotzdem wird deutlich, dass die Gesetzesanforderungen des KJSG auf eine kommunal geprägte, organisatorisch unterschiedliche Praxis treffen, was hinsichtlich der nun anstehenden Umsetzungsbemühungen einige Herausforderungen mit sich bringt.

\section{Der Blick ins Gesetz}

Im Folgenden wird nicht der Versuch unternommen, das Gesetz zur Stärkung von Kindern und Jugendlichen (Kinder- und Jugendstärkungsgesetz - KJSG) in all seinen Facetten zu kommentieren: Zu groß, zu umfangreich, und zu komplex sind die damit verbundenen Änderungen für die Praxis in den Allgemeinen Sozialen Diensten der Jugendämter. Vielmehr erfolgt eine Fokussierung auf einzelne exemplarisch gewählte Teilaspekte. Neben einem Einblick wird hierdurch auch verdeutlicht, dass der ASD ein, wenn nicht sogar der zentrale Akteur in der Umsetzung dieses Artikelgesetzes ist. Der „Erfolg“ des KJSG ist damit auch maßgeblich mit der konkreten Ausgestaltung durch den ASD verbunden. Eine Würdigung der Perspektive dieser Organisationseinheit ist damit von besonderer Bedeutung.

\section{Wird der Kinderschutz verbessert?}

Die Evaluationen problematischer Kinderschutzverläufe beschreiben oft die Schwierigkeiten an den Schnittstellen der beteiligten Professionen und Institutionen. Das KJSG reagiert hierauf mit einer Reihe von Änderungen, wie z. B.:

- Berufsgeheimnisträger_innen, die gem. 4 Abs. 3 KKG Daten an das Jugendamt übermittelt haben, sind künftig in geeigneter Weise an der Gefährdungseinschätzung zu beteiligen ( $\mathbb{8} 8$ a Abs. 1 Satz 2 ).

- In $\$ 4$ Abs. 4 wurde eine Rückmeldeaufforderung für das Jugendamt gegenüber den informierenden Berufsgeheimnisträger_innen - bei Vorliegen einer dringenden Gefahr für das Wohl des Kindes/Jugendlichen, die ein Tätigwerden fordert - verankert. 
- Die Offenbarungsrechte der Berufsgeheimnisträger innen wurden durch die Befugnisnorm in $\mathbb{} 4$ Abs. 3 KKG klarer und eindeutiger benannt.

- Durch $\$ 5$ KKG wurde eine frühzeitigere Mitteilungspflicht für die Strafverfolgungsbehörden eingeführt.

Darüber hinaus wurde in $\mathbb{\$} 8$ a Abs. 4 SGB VIII hinsichtlich der Qualifikation der insoweit erfahrenen Fachkraft ergänzt, dass diese den spezifischen Schutzbedürfnissen von Kindern und Jugendlichen mit Behinderung Rechnung tragen soll. Weitere, verbindliche Qualifikationskriterien für die insoweit erfahrenen Fachkräfte sucht man jedoch auch weiterhin vergebens und eine konkretisierende Profilschärfung für die Funktion der insoweit erfahrenen Fachkraft erfolgt nicht.

Weiter wurde in $\$ 50$ Abs. 2 Satz 2 SGB VIII festgelegt, dass in Verfahren nach den $\mathbb{S}$ 1631b, 1632 Absatz 4, den $\mathbb{S} 1666,1666 \mathrm{a}$ und $\mathbb{} 1682$ BGB sowie in Überprüfungsverfahren hierzu das Jugendamt dem Familiengericht ausgewählte Elemente (Ergebnis der Bedarfsfeststellung sowie entsprechender Überprüfungen, vereinbarte Art der Hilfegewährung sowie hiervon umfasste Leistungen) des Hilfeplans gem. $\$ 36$ Absatz 2 Satz 2 SGB VIII vorlegen muss (in weiteren Sorge- und Umgangsverfahren auf Verlangen). In den ASDs wird derzeit noch gerätselt, wie diese Regelung praktisch umgesetzt werden soll: Die Hilfepläne sind i. d. R. wesentlich umfangreicher als in $\$ 36$ Abs. 2 SGB VIII benannt. So beinhalten sie bspw. die für den Hilfeprozess vereinbarten Ziele, Aspekte der Auftragsklärung und Informationen zu auch vielen persönlichen Belangen der Leistungsberechtigten. Hier stellen sich nun die Fragen, ob die Hilfepläne zukünftig in zwei Teile zu trennen sind, wie lange rückwirkend diese Vorlagepflicht beim Familiengericht greift - vor allem aber auch, welcher Mehrwert aus den im Hilfeplan enthaltenen Informationen für die Familiengerichte in Hinblick auf die bereits bisher vorhandenen Regelungen im \50 Abs. 2 SGB VIII erwächst und welche Auswirkungen diese Änderungen auf die Arbeitsbeziehung mit den Familien haben werden.

In Bezug auf die beiden weiteren, unter der Überschrift "Verbesserungen im Kinderschutz" zu subsumierenden Anwendungsbereiche, die Änderungen im Betriebserlaubnisverfahren ( $\mathbb{S} 45 \mathrm{ff}$. SGB VIII ff.) und die verschärften Kriterien von Auslandsmaßnahmen $(\mathbb{S} 38$ SGB VIII), ist grundsätzliche Zustimmung zu signalisieren. Insbesondere im Hinblick auf problematische Auslandsunterbringungspraktiken vereinzelter Jugendämter in der Vergangenheit war hier eine gesetzgeberische Schärfung notwendig. Gespannt muss man allerdings sein, wie darauf die Anbieterlandschaft reagieren wird. Die Hürden für eine konkrete Unterbringung eines Kin- des oder Jugendlichen im Ausland sind nun nochmals gestiegen und oft auch nicht durch die Jugendhilfeträger beeinflussbar (vor allem die Konsultation der Behörden im EU-Mitgliedsstaatsverfahren), so dass die Zukunft dieser im Einzelfall auch durchaus sinnvollen Unterbringungsform fraglich ist.

Die Veränderungen im Kinderschutz sind weitestgehend zu begrüßen. Entscheidend für einen kommunalen qualitativ guten Kinderschutz wird es nun sein, dass und wie diese einzelnen Themen bestenfalls in kommunalen Gesamtkonzepten zum Kinderschutz zusammengefasst werden (u. a. Stiftung SPI 2021, S. 69). Dazu braucht es neben den Mitarbeiter_innen in den ASDs natürlich auch entsprechende Leitungsressourcen bzw. Stellenanteile im Bereich des Controllings und der Qualitätsentwicklung (vgl. \$ 79 und 79a SGB VIII). Gerade kleinere Jugendämter stehen hier vor höheren strukturellen und konzeptionellen Anforderungen (ebd., S. 78) als größere Organisationeinheiten. Für eine professionelle Weiterentwicklung des Kinderschutzes reicht es folglich nicht aus, „nur das Gesetz zu verändern“.

\section{Müssen wir Beteiligung und Hilfeplanung neu denken?}

Auch in Bezug auf die Hilfeplanung - wie zum Beispiel die Würdigung vorhandener Geschwisterbeziehungen ( $\$ 36$ Abs. 2 SGB VIII) oder die Regelungen zum Übergangsmanagement ( $\$ 36$ b SGB VIII) - sowie die Beteiligung und Beratung ( $\mathbb{S} 8$ und 10a SGB VIII) finden sich im KJSG zahlreiche Änderungen. Der mit dem Bundeskinderschutzgesetz eingeführte elternunabhängige Beratungsanspruch für Kinder und Jugendliche ( $\mathbb{} 8$ Absatz 3 SGB VIII) wird durch das KJSG gestärkt: Er besteht nun uneingeschränkt - also unabhängig vom Vorliegen einer Not- und Konfliktsituation - und ohne Kenntnis der Personensorgeberechtigten. Der Zugang für Kinder und Jugendliche zur Beratung durch das Jugendamt - oder nun auch durch freie Träger - wird hierdurch niedrigschwelliger. Die Betonung, dass die Beteiligung und Beratung in einer von den Kindern und Jugendlichen verständlichen, nachvollziehbaren und wahrnehmbaren Form erfolgt, hebt das zentrale Anliegen hervor, für alle Kinder und Jugendlichen angemessene Beteiligungsformen zu finden. Die nun in $\mathbb{S} 10$ a SGB VIII verankerte Beratung zielt darauf ab, die (potenziellen) Adressat_ innen der Leistungen des SGB VIII in die Lage zu versetzen, eigenverantwortliche Entscheidungen zu treffen und aktiv am Leistungsgeschehen mitzuwirken. Die hier deutlich werdende Achtung und Stärkung der Subjektstellung der Adressat_innen der Kinder- und Jugendhilfe ist dabei ein durchgängiges und zentrales Element des KJSG, das methodisch vor allem in der Verbindung 
von Beratung und Beteiligung umgesetzt wird bzw. werden soll. Eine der programmatischen Zielsetzungen in Bezug auf Kinder und Jugendliche findet sich dabei in $\$ 1$ Abs. 1 SGB VIII: Jeder junge Mensch hat ein Recht auf Förderung seiner Entwicklung und auf Erziehung $z u$ einer selbstbestimmten, eigenverantwortlichen und gemeinschaftsfähigen Persönlichkeit.

Das Recht auf eine selbstbestimmte Persönlichkeit wurde neu in $\mathbb{2}$ Abs. 1 SGB VIII aufgenommen und findet sich auch als eines der zentralen Themen im Bundesteilhabegesetz (BTHG). Was bedeutet aber Selbstbestimmung? Schön und anschaulich fassen dieses Michael Kennedy und Lorin Lewin zusammen: „Selbstbestimmung ist das, worum es im Leben überhaupt geht. Ohne sie kannst $d u$ am Leben sein, aber $d u$ würdest nicht leben, du würdest nur existieren." (Kennedy und Lewin 2004) Selbstbestimmung in diesem Sinne, bedeutet Kontrolle über das eigene Leben zu besitzen, basierend auf Wahlmöglichkeiten zwischen akzeptablen Alternativen. Verwirklicht wird dies im Rahmen der neuen Beteiligungs- und Beratungspflichten, die prozesshaft an zahlreichen Verfahrensstellen verankert sind und die es nun mit Leben zu füllen gilt.

Grundsätzlich sind diese Neuerung und auch Stärkungen bisheriger Regelungen natürlich zu begrüßen. Kritisch diskutiert wird in der Praxis allerdings schon, ob die hier verankerten Handlungsmaximen nicht auch schon vorher fachlicher Anspruch der Arbeit im ASD waren (oder zumindest gewesen sein sollten) und ob diese Neuerungen nun eher „alter Wein in neuen Schläuchen“ sind. Vor diesem Hintergrund wird es besonders wichtig sein - insbesondere auch mit Blick auf eine inklusive Hilfeplanung -, dass die Umsetzung in den ASDs nicht nur mit einem bürokratischen Blick auf neue Verfahrensvorschriften erfolgt, sondern auch zur Reflexion und Weiterentwicklung der übergeordneten Handlungsprinzipien einlädt. Die Soziale Arbeit kann mit der Perspektive auf die Subjektstellung der Adressat_innen auf einen großen Schatz von Theorien, Konzepten und Methoden in der Umsetzung zurückgreifen - entscheidend ist, dass sie dies tut und die entsprechenden Gestaltungs-, Handlungs- und Entscheidungsspielräume hierfür innehat.

\section{Der inklusive Stufenplan}

Der Weg zur Inklusion - als eines der zentralen Anliegen der SGB VIII-Reform - wird in einem Drei-Stufen-Modell beschritten. Das Ganze verpackt unter dem Leitsatz „Hilfen aus einer Hand“. Dabei sollen die Vorstufen dazu dienen, die Kinder- und Jugendhilfe bis 2028 auf eine einheitliche sachliche Zuständigkeit für alle Kinder und Jugendliche unabhängig vom Vorliegen einer Behinderung und der Behinderungsform vorzube- reiten. In Kraft getreten sind dazu z. B. schon die Regelungen zur Übergangsplanung ( $\mathbb{S} 36 \mathrm{~b}$ SGB VIII) oder zur Teilnahme der Jugendämter am Gesamtplanverfah-

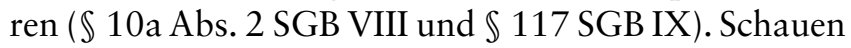
wir exemplarisch etwas konkreter auf die Regelungen zur Übergangsplanung:

\section{Stufe 1: Werden (viele) kleine Lösungen zur großen Lösung?}

Durch die verschiedenen länderweiten Ausführungsgesetze zum Bundesteilhabegesetz ist zwischenzeitlich ein zusätzliches, fast babylonisches Zuständigkeitssystem der Eingliederungshilfe in manchen Bundesländern entstanden. So wanderte zum Beispiel in NordrheinWestfalen die Zuständigkeit für Kinder und Jugendliche mit geistigen und körperlichen Behinderungen in Pflegefamilien erst im Jahr 2020 zum übergeordneten Träger der Sozialhilfe - mit im Gepäck waren Abgrenzungsprobleme und Zuständigkeitsfragen bei Kontakten mit der Herkunftsfamilie, Inobhutnahmen oder $\mathrm{Zu}$ satzleistungen für diese Kinder und Jugendlichen. Bei den Finanzvolumen einer stationären Unterbringung insbesondere auf mehrere Jahre gerechnet - wird die Eingliederungshilfe so zum Kampffeld der verschiedenen Kostenträger. Viele Ressourcen - losgelöst von der eigentlichen Hilfe - müssen hier investiert werden. Das Instrument der Weiterleitung gem. $\mathbb{1 4}$ SGB IX wird dabei auch gerne schon mal dazu genutzt, den Fall trotz klarer Zuständigkeit erst einmal weiterzureichen. Die Folge sind langwierige Kostenerstattungsverfahren und zweit-angegangene Träger, die oft ohne Fallkenntnis Fallentscheidungen treffen müssen.

Mit der durch das KJSG nun in $\$ 36$ b Abs. 2 SGB VIII grundgelegten einseitigen Verpflichtung der Jugendhilfe, bei einem Zuständigkeitsübergang frühzeitig z. B. einen anderen Rehabilitationsträger einzubeziehen (z. B. über die Teilhabeplanung), geht daher ein gewisser Pessimismus einher. Dies vor allem, da für die anderen Systeme keine Kooperationspflicht vorliegt; erst recht nicht die Verpflichtung eine Anschlusshilfe bzw. kooperierende oder ergänzende Hilfe zu leisten. Insofern muss sich in der Praxis zeigen, welche Wirkung diese neue Übergangsregelung entfaltet.

Stufe 2: Jugendamt sucht Verfahrenslots_innen - Stellenanzeige im Jahr 2024

Die zweite Stufe hin zu einer inklusiven Kinder- und Jugendhilfe ist geprägt durch die Einführung von Verfahrenslots_innen gem. $\mathbb{S} 10 \mathrm{~b}$ SGB VIII ab dem Jahr 2024. Diese haben zum einen die Funktion, Leistungsberechtigte durch das Eingliederungshilfeverfahren zu „lotsen“ und unabhängig zu unterstützen. Zum ande- 
ren sollen sie die Jugendämter intern bei der Zusammenführung der Leistungen der Eingliederungshilfe für junge Menschen in deren Zuständigkeit unterstützen und halbjährlich gegenüber dem öffentlichen Träger zu Erfahrungen mit anderen Rehabilitationsträgern, auf struktureller Ebene etc. berichten. Befristet ist diese vom öffentlichen Träger zu erbringende Leistung bis zum 31.12.2027. Obwohl die Zielsetzung dieses Aufgabenprofils klar ist, stellen sich in der Praxis Fragen, die bis zur Einführung der Verfahrenslots_innen geklärt sein müssten. So zum Beispiel:

- Gehören die benannten Aufgaben nicht eigentlich zum Aufgabenprofil des ASD oder eines Spezialteams Eingliederungshilfe?

- Wie ist in Konfliktfällen zu verfahren?

- Wer ist erstzuständig, bearbeitet den „Fall“ also zuerst?

- Welche Fachkräfte mit welcher Qualifikation sind für diese Aufgabe geeignet und woher kommen diese?

Zur Klärung dieser Themen sind der Gesetzgeber und der überörtliche Jugendhilfeträger gefragt. Darüber hinaus wäre eine Präzisierung des Aufgabenprofils insbesondere vor dem Hintergrund der unterschiedlichen kommunalen Organisationsprofile wünschenswert. Ansonsten steht zu befürchten, dass die zentrale Rolle, die den Lots_innen bis zum Jahr 2028 und zur Realisierung der „Große Lösung“ zugeschrieben wird, nicht ausgefüllt werden kann.

\section{Stufe 3: Jugendamt sucht neues Gebäude - Anzeige im Jahr 2028?}

Das Ziel „Hilfen aus einer Hand“ und damit die einheitliche sachliche Zuständigkeit der öffentlichen Jugendhilfe für alle Kinder und Jugendliche scheint gewaltig: Die Jugendämter müssen mit vielfachen neuen Zuständigkeiten, zusätzlich benötigten finanziellen und personellen Ressourcen aber auch mit zusätzlichen Anforderungen rechnen. Bis dahin gilt es aber von Politik und Praxis noch viele Fragen zu beantworten und Unsicherheiten aufzulösen. Spannend wird dabei vor allem sein, wie das zukünftige Leistungsgesetz ( $\mathbb{1 0}$ Abs. 4 S. 2 SGB VIII) den Leistungstatbestand und den Behinderungsbegriff definiert oder wie der Bruch zwischen einer objektiv-funktionalistischen Bedarfsfeststellung und der partizipatorischen dynamischen Bedarfsklärung überwunden werden soll bzw. kann (Hopmann et al. 2020, S. 343 ff.). Im Interesse der Kinder, Jugendlichen und Familien - aber auch der Fachkräfte vor Ort - ist es wichtig, die Deutungs- und Entscheidungshoheit nicht der Politik oder den Verbänden zu überlassen. Vielmehr ist die kommunale Kinder- und Jugendhilfe aufgefor- dert, sich frühzeitig und aktiv in diesen Prozess einzubringen und diesen mitzugestalten. $\mathrm{Zu}$ groß sind ansonsten die Risiken und Folgen eines „Aussitzens und Abwartens"!

\section{Ein kleines Resümee}

Selbst wenn im Rahmen dieses Beitrags nur Teilbereiches des Kinder- und Jugendstärkungsgesetzes exemplarisch aufgegriffen werden konnten, wird deutlich, dass „ein Powerpoint-Vortrag im Team“ nicht als zentraler Umsetzungsimpuls in den Jugendämtern ausreichen wird. Zahlreiche Regelungen führen zu Komplexitätserweiterungen, eine Vielzahl an Details sind noch unklar und müssen zunächst durch die kommunale Praxis erprobt und gefüllt werden. Die Herausforderung in Anbetracht der gegenwärtigen Rahmenbedingungen ist dabei groß: Für viele wird es eine enorme Kraftanstrengung sein, den Zielsetzungen dieses Gesetzes gerecht zu werden. Insgesamt können zahlreiche der mit der Einführung des KJSG aber verbundene Änderungen aus ASD-Perspektive befürwortet und als wichtig unterstrichen werden. Einige andere bringen nicht zu unterschätzende Nebenwirkungen mit sich und wieder andere sind durchaus auch kritisch zu bewerten. Im Hinblick auf die Realisierung und auch Weiterentwicklung dieser vielfältigen Themen vor Ort scheint es insbesondere bedeutungsvoll, das Bewusstsein dafür zu schärfen, dass diese (neuen) Anforderungen auf eine unterschiedliche Praxis treffen und auch deren Umsetzung regional differenziert und unterschiedlich erfolgen wird.

Das, was aber allerorts gleich sein wird, ist ein (weiterhin) zunehmender Personalbedarf. Personal, das zum einen immer schwieriger zu finden und zum anderen immer schwieriger zu halten ist. Der zunehmende „Kampf ums Personal“ wird daher auch eine Sekundärfolge dieser Reform sein - zumal da z. B. durch den Rechtsanspruch auf Ganztagsbetreuung ab 2026 auch in anderen Bereichen der Kinder- und Jugendhilfe weitere Personalbedarfe zu erwarten sind. Vor diesem Hintergrund kommt der nun in $\mathbb{S} 79$ Abs. 3 SGB VIII verankerten Verpflichtung zur Nutzung eines Personalbemessungsverfahrens eine besondere Bedeutung zu. Dies löst das Problem aber keinesfalls - vielmehr sind die ASDs gefordert, bereits vor und während des Studiums das Interesse an der Arbeit im ASD zu wecken, Studierende gut zu begleiten und die Berufseinmündung professionalitätsfördernd zu gestalten (vgl. BAG ASD 2021). Darüber hinaus bringen die gesetzlichen Veränderungen auch Qualifizierungsbedarfe für die langjährigen Mitarbeitenden im ASD mit sich, beispielsweise im Hinblick auf die Weiterentwicklung der Beratungs- und Hilfeplanungskompetenzen. 


\section{Kann der ASD also Kinder- und Jugendstärkungs- gesetz?}

Grundsätzlich ja! Um den mit dem KJSG verbundenen großen Erwartungen aber gerecht werden zu können, braucht es nun vor allen den Willen zur Realisierung der darin liegenden Potenziale, die Offenheit zur Diskussion und Erörterung der damit einhergehenden Hürden und Herausforderungen und die Bereitschaft, die Dinge kontinuierlich anzugehen und voranzubringen. Die Erwartungen an die örtliche Jugendhilfe sind berechtigter Weise groß. Um diesen gerecht werden zu können, sind gemeinsame Kraftanstrengungen aller Akteur_innen im Feld erforderlich: Von Hochschulen zur Praxis, von den Landesjugendämtern zu den Jugendämtern, von Leitungsebenen zu Mitarbeiter_innen und von den örtlichen Jugendämtern in die Kommunen hinein. Der ASD als zentraler Basisdienst der Kinder- und Jugendhilfe sollte das im KJSG liegende Potenzial jedenfalls aktiv und konstruktiv nutzen und seinen wichtigen Beitrag zur professionellen Weiterentwicklung der Kinder- und Jugendhilfe leisten!

Eingegangen. 15. September 2021

Angenommen. 20. September 2021

Funding. Open Access funding enabled and organized by Projekt DEAL.

Open Access. Dieser Artikel wird unter der Creative Commons Namensnennung 4.0 International Lizenz veröffentlicht, welche die Nutzung, Vervielfältigung, Bearbeitung, Verbreitung und Wiedergabe in jeglichem Medium und Format erlaubt, sofern Sie den/die ursprünglichen Autor(en) und die Quelle ordnungsgemäß nennen, einen Link zur Creative Commons Lizenz beifügen und angeben, ob Änderungen vorgenommen wurden.

Die in diesem Artikel enthaltenen Bilder und sonstiges Drittmaterial unterliegen ebenfalls der genannten Creative Commons Lizenz, sofern sich aus der Abbildungslegende nichts anderes ergibt. Sofern das betreffende Material nicht unter der genannten Creative Commons Lizenz steht und die betreffende Handlung nicht nach gesetzlichen Vorschriften erlaubt ist, ist für die oben aufgeführten Weiterverwendungen des Materials die Einwilligung des jeweiligen Rechteinhabers einzuholen.

Weitere Details zur Lizenz entnehmen Sie bitte der Lizenzinformation auf http://creativecommons.org/licenses/by/4.0/deed.de.

\section{Literatur}

BAG ASD (2021). Qualifizierte Fachkräfte für den ASD - gemeinsame Verantwortung stärken! Ein Positions- und Diskussionspapier der BAG ASD mit Vertreter*innen aus Hochschulen NRWs zur Anregung weiterer Dialoge. https://www.bag-asd.de/positionspapier-zum-projekt-qualifiziertefachkraefte-fuer-den-asd/. Zugegriffen: 13. Sept. 2021.

Bundesarbeitsgemeinschaft der Landesjugendämter, \& Müller, H. (2020). Der Jugendamts-Monitor: Aufgaben - Trends - Daten. Mainz: Bundesarbeitsgemeinschaft der Landesjugendämter.
Bundesministerium für Frauen, Senioren, Familie und Jugend (BMFSFJ) (2021a). Pressemitteilung: Kinder und Jugendliche in schwierigen Lebenslagen stärken. https://familienportal.de/familienportal/meta/aktuelles/aktuelle-meldungen/kinder-und-jugendliche-in-schwierigen-lebenslagen-staerken-179210. Zugegriffen: 12. Sept. 2021.

Bundesministerium für Frauen, Senioren, Familie und Jugend (BMFSFJ) (Hrsg) (2021b). Ergebnisbericht der wissenschaftlichen Begleitung zum Dialogprozess „Mitreden - Mitgestalten: Die Zukunft der Kinder- und Jugendhilfe“". https://ikj-mainz.de/wp-content/uploads/sites/3/2020/10/IKJ-Ergebnisbericht-Wiss.-Begleitung_gesamt.pdf. Zugegriffen: 12. Sept. 2021.

Hopmann, B., Rohrmann, A., Schröer, W., \& Urban-Stahl, U. (2020). SGB VIII Reform: Quo vadis Hilfe- und Teilhabeplanung mit jungen Menschen und ihren Eltern? JAmt, 93(7-8), 338-346.

Kennedy, M., \& Lewin, L. (2004). Was ist Selbstbestimmung und was nicht. http://bidok.uibk.ac.at/library/kennedy-selbstbestimmung.html. Zugegriffen: 12. Sept. 2021.

Stiftung SPI (2021). Gutachten zu Organisation, Struktur, Größe, Standards, Qualität, Fortbildung und Weiterbildung in nordrhein-westfälischen Jugendämtern. Abschlussbericht. https://www.landtag.nrw.de/portal/ WWW/dokumentenarchiv/Dokument/MMI17-316.pdf. Zugegriffen: 17. Sept. 2021. 